\title{
Analysis and Testing of Mobile Wireless Networks
}

\author{
Richard Alena, Darin Evenson*, Victor Rundquist $\dagger$ \\ NASA Ames Research Center \\ Moffett Field, CA 94035-1000 \\ (650) 604-0262 \\ ralena@mail.arc.nasa.gov \\ * Naval Postgraduate School, daevenso@nps.navy mil \\ † DeAnza College, vrundquist@mail.arc.nasa.gov
}

\begin{abstract}
Wireless networks are being used to connect mobile computing elements in more applications as the technology matures. There are now miany products (such as 802.11 and $802.11 \mathrm{~b}$ ) which run in the ISM frequency band and comply with wireless network standards. They are being used increasingly to link mobile Intranet into wired networks. Standard methods of analyzing and testing their performance and compatibility are needed to determine the limits of the technology.
\end{abstract}

This paper presents analytical and experimental methods of determining network throughput, range and coverage, and interference sources. Both radio frequency (RF) domain and network domain analysis have been applied to determine wireless network throughput and range in the outdoor environment. Comparison of field test data taken under optimal conditions, with performance predicted from $\mathrm{RF}$ analysis, yielded quantitative results applicable to future designs.

Layering multiple wireless network subnets can increase performance. Wireless network components can be set to different radio frequency-hopping sequences or spreading functions, allowing more than one subnet to coexist. Therefore, we ran multiple 802.11-compliant systems concurrently in the same geographical area to determine interference effects and scalability. The results can be used to design of more robust networks which have multiple layers of wireless data communication paths and provide increased throughput overall.

Copyright 2002 by the Institute of Electrical and Electronics Engineers. No copyright is asserted in the United States under Title 17, U.S. Code. The U. S. Government has royalty-free license to exercise all rights under the copyright claimed herein for government purposes. All other rights are reserved by the copyright owner.

Paper 410 .

\section{TABLE OF CONTENTS}

\author{
Abstract \\ Introduction \\ Wireless Network Technologies \\ Mobile Exploration System Overview \\ Wireless Network Metrics \\ RF Propagation Theory \\ MEX Testbed Data \\ RF Link Analysis \\ Interference And Scalability \\ Conclusions \\ References
}




\section{INTRODUCTION}

By nature, wireless networks differ from wired networks by the transient, ad-hoc connections they establish. The term wireless local area network (WLAN) represents a communications method or system established through the use of radio frequency (RF) technology that can function either as an extension to an existing local area network (LAN) or as an alternative for a wired LAN. Relying on complex spread-spectrum radio signals, the bandwidth and connection quality vary dramatically dependant upon free space propagation characteristics. The networks are increasingly used to connect mobile computing elements in indoor and outdoor environments, covering distances ranging from several meters to several kilometers and providing bandwidths from hundreds of kilobits per second to several megabits per second.[1]

The Intelligent Mobile Technologies (IMT) team at NASA Ames Research Center studied the integration of disparate collections of mobile nodes consisting of sensors, notebook computers, servers, graphics tablets, and display devices using both 802.11 and $802.11 \mathrm{~b}$ wireless network products. [2] The team developed a pragmatic approach to testing and defining the characteristics of these products consisting of mathematical modeling, network performance metrics, radio frequency (RF) domain analysis and field testing. [3] The metrics show sustainable and peak network throughput, range and coverage results which can be correlated with interference effects. Related to interference is scalability, where non-interfering systems can be layered to provide a much greater aggregate bandwidth capability.

This paper presents a brief overview of WLAN technologies, analysis methods, performance testing and field test results. An introduction to RF propagation theory and a simple model of the access protocol provide some interesting background for interpreting the results. The goal of this effort is to provide performance data relevant to the design and application of next-generation WLAN systems.

\section{WIRELESS NETWORK TECHNOLOGIES}

There are several technologies that are used to create WLANs, including frequency-hopping spread-spectrum (FHSS) and direct-sequence spread-spectrum (DSSS) communications. A discussion of spread-spectrum technologies is important for understanding the characteristics of WLANs and the difference in test results. [4] Spreadspectrum communications were developed during the late 1940 's as a mechanism to provide a reliable and secure communications method for the military under combat conditions. [5] Spread-specirum is the major technology of choice in the commercial WLAN industry because many devices can be used simulkaneously with minor interference concerns. With the use of spread-spectrum commun- ications, more bandwidth is used than in conventional narrowband transmission that is based on a specific radio frequency. This results in an inefficient use of bandwidth, but it mitigates the risks of staying on one frequency and being intercepted or jammed. Because the signal is spread over a greater frequency spectrum, the receiver must know the parameters of the original signal in order to recover it correctly. If the receiver is not tuned to the correct frequency at the correct time, the signal will appear as background noise.

With frequency-hopping spread-spectrum (FHSS) communications, a narrowband carrier radio frequency is shifted in discrete increments of frequency, predetermined by a code. The amplitude, and thus the power and energy in each hop are constant. The frequency shift is based on a pattern generated from a code called the hopping sequence that spreads transmission over a wide frequency band. For the signal to be received correctly, the hopping sequence must be known in advance by both the receiver and the transmitter. With direct-sequence spread-spectrum communications (DSSS), a narrowband carrier radio frequency is modulated by a digital "chipping code" with the code bit rate being larger than the information bit rate thus spreading the signal over a very large bandwidth. Because the "chipping code" adds redundancy to the information being transmitted, this permits a receiver to recover the original data even if one or more bits are damaged during transmission. Because the DSSS technique spreads information over a wide bandwidth, a receiver without the correct "chipping code" views the signal as low power wideband noise.

Compliance with standards ensures appliance interoperability with other WLAN components from different vendors that have adopted the same standard. [6] In the United States, the FCC governs radio transmissions, and most WLANs broadcast over one of the Instrumentation, Scientific, and Medical (ISM) bands. These include 902$928 \mathrm{MHz}, 2.4-2.483 \mathrm{GHz}, 5.15-5.35 \mathrm{GHz}$, and 5.725$5.875 \mathrm{GHz}$. In 1997 , when the first internationally recognized IEEE 802.11 standard arrived, there was a sudden surge of market interest, resulting in the advent of higher speeds with $802.11 \mathrm{~b}$ and $802.11 \mathrm{a}$. The 802.11 standard refers to the media access control (MAC) layer of the ISO network model and also defines modulation type and data rates. Quickly summarizing, 802.11 defines a $2 \mathrm{Mbit} / \mathrm{sec}$ data rate, and $802.11 \mathrm{~b}$ defines an $11 \mathrm{Mbit} / \mathrm{sec}$ data rate in the ISM frequency band. This study tested both types of WLAN systems.

In a typical WLAN configuration, a transmitter and receiver, or transceiver device, called an access point (AP), connects to the wired network from a fixed location using standard cabling. At a minimum, the AP receives, buffers and transmits data between the WLAN and the wired network infrastructure. A single AP can support a 
small group of users and functions within a range of less than one hundred to several hundred feet. The access point may be mounted anywhere that is practical as long as the desired radio coverage is obtained. The AP provides a single cell or subnet of WLAN coverage. Multiple APs provide multiple subnets, allowing mobile users to roam from one subnet to another maintaining connection to the network, much like a cellular phone system.

To solve particular problems of WLAN topology, the network designer might choose to use an extension point (EP) to augment the network of APs. EPs (or repeaters) extend the range of the network by relaying radio signals from an AP to a station or another EP. Extension points may be strung together in order to pass along messaging from an AP to far-flung clients. One last item of WLAN equipment to consider is the directional antenna. Directional antennas increase the gain, or relative focus, of the antenna which increases the range and the reliability of a given signal. The other option to increase range would be to substantially increase power output, but this is not always desirable in a military environment where tactical operators are focused on clandestine operations. It is also a concern in mobile applications, where minimum power consumption is desired.

\section{MOBILE EXPLORATION SYSTEM OVERVIEW}

The Mobile Exploration System (MEX) is a testbed for developing communication system architecture for planetary exploration. The MEX is a project of the
Human Exploration and Development of Space (HEDS) Enterprise at NASA. [7] The testbed is used for technology development of computational components, communications systems, and collaborative applications. The MEX is used for developing operations scenarios for planetary exploration and is being tested through a series of field trials. Recently the MEX was taken to the NASA Haughton Mars Project (HMP), a Mars analog field research site located in the Canadian High Arctic, an extremely remote and hostile environment, one that was chosen for its similarity to the planet Mars. [8] The MEX is assembled from commercial technologies, and combines characteristics of both satellite and surface communication systems. Satellite communications are for the long haul connections between major nodes, such as the planetary Base to Mission Control on earth. [9] The planetary surface communications are designed for high bandwidth, low latency connections for operations conducted for exploration. The nodes are compact and low power, and support many special purpose nodes with ancillary functions such as position estimation, imaging, data collection and environmental monitoring. The surface systems of the MEX are the focus of the tests conducted for this paper. [10]

The MEX communication systems include Proxim's RangeLAN2 radios for WLAN implementation. A second WLAN from the Orinoco product line was incorporated into the MEX architecture this year, representing an $802.11 \mathrm{~b}$ product. The computers all ran Microsoft Windows operating systems, and all communications occurred in the unlicensed 2.4 Ghz ISM band.

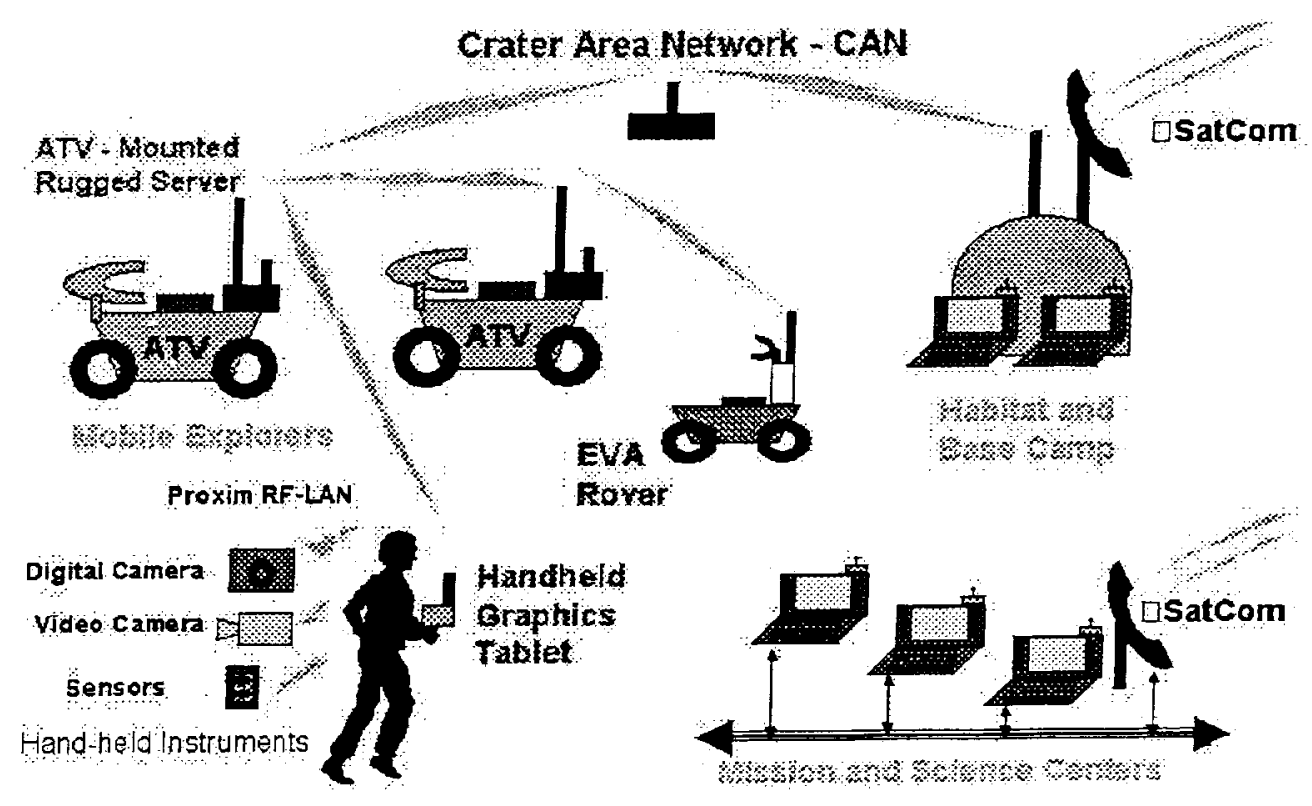

Figure 1. Mobile exploration system, 2001 
The primary goal was to characterize and compare the performance of the two WLANs in field conditions. Both products are actually designed for indoor short-range communications and were adapted to the outdoor environment and longer range communications through the use of environmental enclosures and high-gain directional antennas. An overview of the MEX architecture and its individual components ranging from handheld devices and small sensors, to ruggedized servers mounted on an all terrain vehicle (ATV) is shown in Figure 1 .

The WLAN components originally tested are from Proxim, Inc., which was the first-to-market in 1994 with its RangeLAN2 products operating in the $2.4 \mathrm{Ghz}$ frequency band. At that time, Proxim's OpenAir protocol was proprietary and there was no WLAN standard. Proxim was part of the committee specifying the proposed IEEE 802.11 protocol, and soon after the release of the standard, Proxim adopted it. However they also had a huge market share based on the OpenAir protocol and decided to support both product lines. The RangeLAN2 WLAN components tested operate according to the OpenAir specification at data rates up to $1.6 \mathrm{Mbps}$, but, given their historical contribution to the 802.11 protocol, are directly comparable to 802.11 products operating at 2.0 Mbps. Figure 2 shows some of the testbed components in the field.

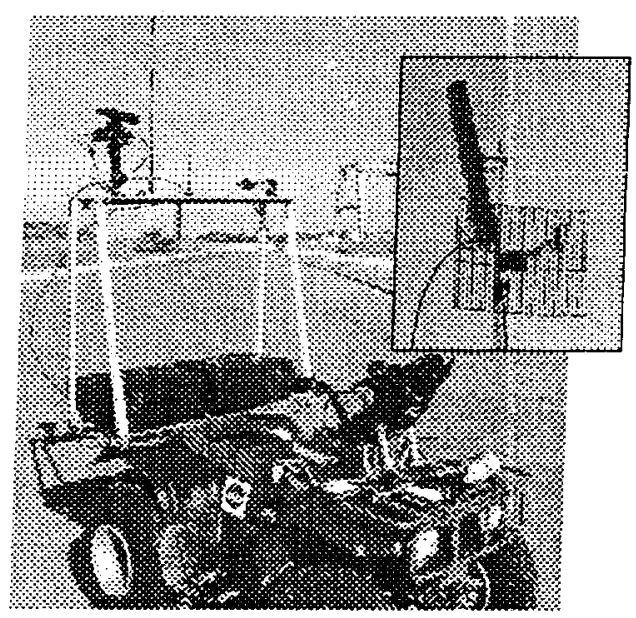

Figure 2. MEX components

The MEX operational simulations have demonstrated the need for shorter latencies and more sustainable bandwidth. To accompish this, a leading $802.11 \mathrm{~b}$ technology based on the Orinoco (formerly Lucent WaveLAN) product was tested and compared to the Proxim product. The MEX architecture made use of all the WLAN configurations noted. Peer-to-peer networks are utilized with some of the handheld mobile devices, with APs at base and EPs functioning as repeaters located on high hills. Directional antennas are used to increase the range and connectivity of the MEX wireless technologies.

\section{WIRELESS NETWORK METRICS}

The physical characteristics that influence WLAN performance can be measured using a few key parameters. "Range" is the distance over which a WLAN can communicate and is a function of transmitted power and receiver sensitivity, antennas used and propagation loss. Interactions with typical building objects, including walls, metal and even people, can affect how energy propagates and thus what range a particular system achieves. The range for typical WLAN systems varies from under 100 feet to more than 20 miles. "Coverage" is the geometrical distribution of link viability and is given as a set of angles in three dimensions and is generally determined by antenna choice. Range can be extended by increasing power or by using directional antennas or repeaters.

"Throughput" is the sustainable data rate through a WLAN given in bits per second (bps) or bytes per second. Peak and raw data rates are related measures. Factors that affect throughput include the number of users, MAC layer effects, propagation loss, and multipath and other interference sources. A complementary measurement is latency, the time lag for a packet to be transmitted over the WLAN, which can range from several milliseconds to seconds. Generally, latency can be highly variable even under constant conditions. Also, latency and throughput are inversely proportional.

The "unlicensed" nature of radio-based WLANs means that other products that transmit energy in the same frequency spectrum can interfere with a WLAN system. Microwave ovens are a concern in the $2.4 \mathrm{Ghz}$ range. Another concern is co-locating multiple WLANs. While WLANs from some manufacturers interfere with other WLANs in the same frequency bands, others coexist without interference. Often this is an issue of network domain and channel management practices. Interference is measured as a decrease in throughput, range and coverage or as an increase in latency and can be confirmed by varying the interference source or by using a spectrum analyzer.

Wireless networks can be scaled in two ways: by increasing the number of nodes of a given WLAN or by layering more WLANs in the same coverage area. Interference effects limit layering. Scalability can be expressed by a graph of aggregate throughput versus number of nodes or by aggregate throughput versus number of WLAN subnets. Due to complex frequency domain interactions, this must be determined using physical testing of multiple subnets.

A variety of network modeling tools can be applied to help simulate performance of candidate architectures, taking into account the complex timing of network data transfers. Extend, developed by Imagine That, Inc., is an 
advanced simulation tool designed to develop dynamic models for real processes. Extend allows blocks to be placed in hierarchical structures to be simplified and utilized in other parts of the model. For example, transmission delay is a characteristic that is used throughout the model, and is therefore placed in a hierarchical block for use in multiple locations throughout the model.

Evenson modeled the WLAN at the Media Access Control (MAC) layer of IEEE 802.11 that uses the CarrierSense Multiple-Access Collision Avoidance scheme.[11] It specifies that the transmitter must send out a very short packet called a "request to send" (RTS) before actually transmitting the data packet. Only the destination receiver can respond to the RTS with a "clear to send" (CTS). All other stations must stop transmitting until the data packet is sent and an acknowledgment (ACK) is received. The three types of message traffic modeled were the RTS, CTS, and data packets. The basic component of the Extend MAC model is the modeling of transmission delay. Transmission delay was modeled by dividing the message size by the respective link bandwidth to represent the delay encountered in sending data over the network. The MEX architecture was modeled using these building blocks to determine latency variations.

One goal of the modeling was to determine the MAC layer effect on latency. Many iterations of the model were nun to see the effects of changing message size and bandwidth. The model would generate plots representing the adjustment of the network to the traffic generated by multiple nodes with similar message profiles. As shown in Figure 3, the latency increases in the beginning of the simulated 10 -second run, as the network is loaded with traffic. As time progresses, however, latency varies as the network adjusts to the traffic load coming from each node. Due to the functioning of the 802.11 MAC protocol, the network adjusts to smooth out the effects of initial latency increases. Notice that there are two sustainable modes of data transfer in the steady state, one with lower average latency than the other.

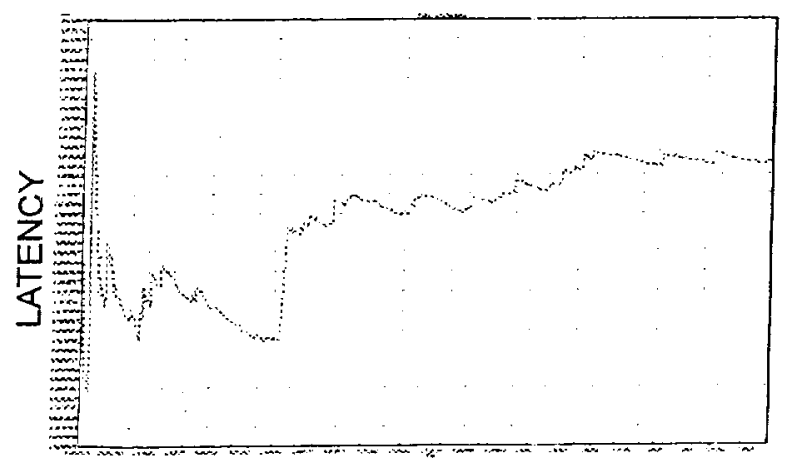

Figure 3. Plot of latency varying by message size over simulation time.

\section{RF PROPAGATION THEORY}

A radio system transmits signals through an antenna, forming a propagating electromagnetic wave that is received by a complementary antenna and radio.[12] The maximum distance between a RF transmitter and receiver depends on several system and path variables including transmitter output power, receiver sensitivity and noise threshold, antenna design, and propagation loss. Terrain and atmospheric conditions can affect propagation loss. Ideally, isotropic antennas have equal radiation or reception of power in all directions. Practically, the closest to this is an omni-directional dipole antenna, which spreads the majority of radiated power in a 360 degree doughnut-shaped belt known as a "main lobe." Directional antennas shape or focus the main lobe energy in such a way that the majority of it is radiated in a specific solid angle. "Antenna gain" is a measure of the directivity of an antenna. It is defined as the ratio of the radiation intensity in a given direction to the radiation intensity that would be obtained if the same power were to be radiated isotropically. The "radiation pattern" of an antenna is a graphical representation in either polar or rectangular coordinates of the spatial energy distribution of an antenna. "Antenna beamwidth" is defined by the angle that subtends the two half-power points $(-3 \mathrm{~dB})$ on either side of a directional antenna's main lobe radiation pattern.

A concern of WLAN design is the loss the carrier signal will suffer as it travels to the receiving station. Study of this consideration is called "link analysis." Immediate loss is due to the attenuation or "spreading loss" affecting any transmitted signal, also known as "free space path loss" (Lfs). By estimating Lfs when designing the link, radio unit capabilities can be estimated to ensure a certain minimum signal threshold for the receiver. Lfs is a function of the square of the distance from the transmitter, and is shown in the following equation.

$$
L_{f s}=\left(\frac{4 \pi d}{\lambda}\right)^{2}
$$

where $d=$ distance, $\lambda=$ wavelength

Radio waves suffer intermittent additional losses in signal strength through absorption as they travel through the atmosphere. These intermittent losses, the "fade margin" in link analysis, may account for additional signal degradation. The total loss for a link can be better estimated once free space and fade margin losses are accounted for. (Incidental losses in cables and connectors can be addressed as necessary.) Total path loss is then used to estimate power received $(\mathrm{Pr})$ at a certain distance. This knowledge will enable engineers to determine how radiation requirements, antenna specifications and placement, and cell coverage will affect the prescribed coverage and performance objectives. 
RF link analysis determines the difference between the nominal output power of a transmitter and the minimum input power required by a receiver. For a link to provide a certain performance objective, the transmitted power and system gains must be greater than all the losses that impinge on the carrier signal as it propagates the distance, and those losses resident in the end system's internal circuitry. This balancing of gains and losses is often referred to as a "link budget." Link budgets predict the reliability of a communication path given particular system parameters. A link budget can be expressed by the Friis equation:

$$
P_{r(d B)}=P_{t}+G_{t}-L_{f s}-L_{x}+G_{r}
$$

where $P_{r}=$ power at the receiver $(\mathrm{dBm})$

$$
\begin{aligned}
& P_{t}=\text { transmitter output power }(\mathrm{dBm}) \\
& L_{f s}=\text { free space path loss }(\mathrm{dB}) \\
& \left.L_{x}=\text { extra losses (e.g. multipath }\right)(\mathrm{dB}) \\
& G_{t}=\text { transmit antenna gain }(\mathrm{dB}) \\
& G_{r}=\text { receive antenna gain }(\mathrm{dB})
\end{aligned}
$$

\section{MEX TESTBED DATA}

The Proxim system field tests were focused on determining calibration curves for latency and throughput versus distance using different combinations of omni-directional and directional antennas. The antennis' had varying performance characteristics included beamwidth and gain. The goal was to find the optimal anterna combination for the intended operational environment. Range and coverage areas for each system will help define further field simulations.

Procedures were developed based on the diagnostic tools available from the radio manufacturers and methods developed by the IMT lab.[14] The Proxim WLAN components have internal radio diagnostic tools to test parameters such as throughput and latency through a series of broadcast and directed site surveys. In broadcast site surveys, the active nodes of the network were discovered by sending out packets to all nodes within antenna range, and waiting for responses from all nodes that receive the signal. Once the network topology was established and proper operation of the network verified, performance information on individual links was found through directed site surveys between individual nodes, based on the MAC address.
Figure 4 shows the basic set up for network testing. Communications testing consisted of placing the access point (AP) at a fixed base location and running a series of directed site surveys to the mobile node EP mounted on the ATV. Several runs of latency and throughput data were collected, and then the distance to the AP was doubled. From this, range calibration curves were generated for various radio and antenna combinations.

Our Ames Research Center test site provided a total distance of $1.1 \mathrm{~km}$ clear line-of-sight with no obstructions, no obvious sources of multipath or interference, and no variations in elevation. The antennas were mounted 6 feet above the ground at the same exact level at each end. Extraneous sources of RF interference had to be eliminated, and to insure this a spectrum analyzer was used to measure RF energy in the $2.4 \mathrm{Ghz}$ band. The spectrum analyzer, which delivers a reasonable graph of relative $R F$ energy, was a sampling type provided as a utility with the Proxim cards. Test points were started at $10 \mathrm{~m}$ and doubled in distance at each test point up to the maximum $1.1 \mathrm{~km}$ distance.

Each antenna combination simulated a "backbone" link of the MEX architecture such as the primary repeater to mobile ATV link. The following antenna combinations were tested for the Proxim system during this research:

- $0 \mathrm{~dB}$ omni-directional to $0 \mathrm{~dB}$ omni-directional

- $11 \mathrm{~dB}$ planar sectoral to $0 \mathrm{~dB}$ omni-directional

- $24 \mathrm{~dB}$ directional parabolic to $0 \mathrm{~dB}$ omni-directional

The Proxim radios used for these tests were of the OpenAir XR series which provides $500 \mathrm{~mW}$ output power. They are FHSS types with a raw data rate of $1.6 \mathrm{Mbps}$. The Orinoco radios provide $32 \mathrm{~mW}$ output power and are 802.11 b DSSS type with a raw data rate of $11 \mathrm{Mbps}$. The radio automatically controls transmission power and speed. Both radios fall back to lower data rates if link margins deteriorate. What are the performance tradeoffs between these two different systems measured in the field under ideal conditions and how does this relate to RF link analysis?

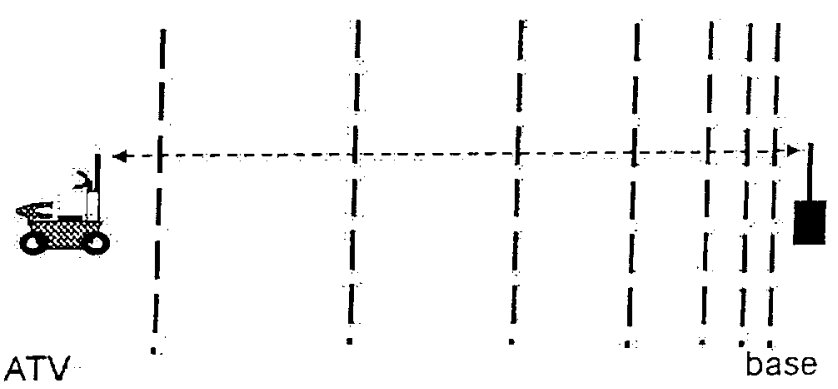

Figure 4. MEX field test setup 
For the Orinoco system, high-level network throughput measurements were made using large file transfers (3 $\mathrm{MB})$ through the WLAN. Application-level sustainable network throughput was determined using a TCP/IP file transfer via FTP: an FTP server was set up at the base AP, the mobile ATV computer requested a file from the server, and the file was transferred. File size and time to download were recorded, and average sustainable network throughput obtained by dividing file size by time. The test was then reversed and the same file uploaded to the server. This is done because the choice of antennas and radio characteristics can result in an asymmetrical connection.

The Orinoco by Agere Systems product was tested with only the built-in antennas because there were no appropriate external antenna connections for it at the time of the tests. Test tools provided by Agere systems give RF domain signal levels and network throughput data simultaneously. The antenna is a slot dipole with about $0 \mathrm{db}$ gain It is notable that that the built-in antenna has a unique dispersion pattern resembling a horizontal flat half-torus. This was experimentally determined by placing the radios at a distance and measuring the radio-level data collected while changing the orientation of one antenna in relation to the other. FTP throughput and RF signal level data were collected in the same manner as the Proxim system.

Finally, formal link analysis of the two systems' RF characteristics was done using manufacturers' specifications. The expected range in a vacuum was compared with the actual range achieved in air (with water vapor) and additional factors such as atmospheric absorption and parasitic losses calculated.

The performance of future wireless network designs can be predicted using this analysis method, and the testbed data was essential for determining these correction constants in the link analysis. The first important results are the Proxim calibration charts for throughput and latency established for the individual antenna combinations. Figure 5. shows throughput of data on tested link in Kbits per second. The generally decreasing throughput at increasing distances on each test set was expected and in accordance with theory. Also, the higher the antenna gain the higher the throughput and the larger the effective distance for communication. These calibration curves are the result of numerous data runs and represent averages from several runs. However, there is an unexpected dip at midrange, when throughput varied about a factor of two. At the closest test distance of $10 \mathrm{~m}$, there was highest throughput and lowest latency, as expected, followed by a significant drop in throughput at $20 \mathrm{~m}$. There is a corresponding increase in latency (shown in Figure 6) that may explain the throughput loss. This dip occurs at too short a distance to be the result of free space losses and low signal strength. It should be noted that the RF signal strength between the two radios varies significantly with each antenna combination, and yet this dip occurs at nearly the same distance for each graph. Could this be a MAC layer timing interaction?

Figure 6 shows average latency values for the series of packets sent during the directed site survey at each distance. Latency and throughput are inversely related, and this is evident in the data. To test the latency, the computer sends packets for ten seconds or other selected interval and monitors the average, maximum and standard deviation of the latency of the packets. The packets are sent at maximum rate and therefore also provide the throughput numbers. This is all measured using the radio diagnostic tools that come with the Proxim product. The latency increase and throughput decrease at $20 \mathrm{~m}$ has a possible explanation. The OpenAir protocol was designed based on expected use in a commercial environment, typically inside a building. Remembering the RTS/CTS construct previously discussed, it is possible that the protocol simply does not function optimally at a range of $20 \mathrm{~m}$.

\section{Proxim Throughput}

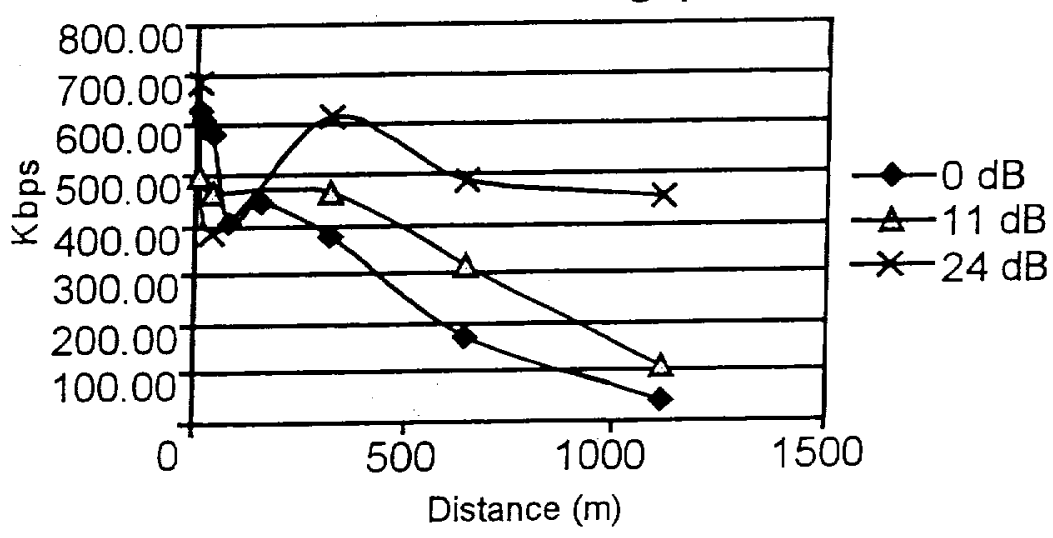

Figure 5. Proxim throughput calibration curves. 


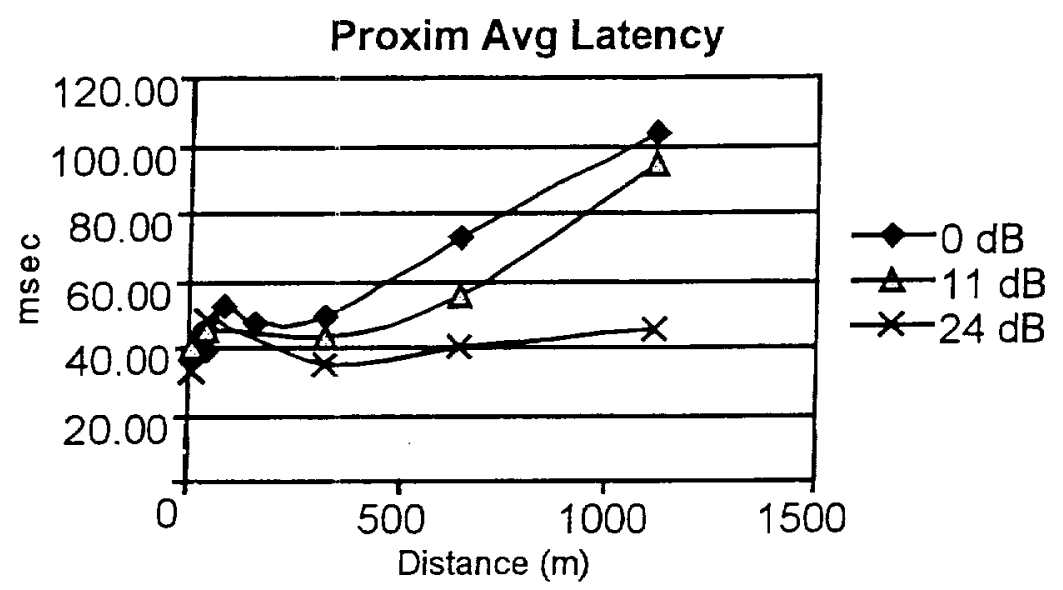

Figure 6. Proxim latency calibration curves

The interaction at the MAC layer could have been optimized for use within an average office space which is rarely larger than $10 \mathrm{~m}$ square The Extend MAC layer model showed considerable variations in throughput early in the simulation and at least two modes of sustainable throughput. These variations cannot be the result of $R F$ signal strength variations, but may be the result of interactions of the MAC layer coinciding with the variation of free-space propagation times. This is only a hypothesis, but simple modeling of the MAC layer did demonstrate that it is possible.

After this initial variance, throughput and latency were well behaved. Throughput increases significantly beyond the initial dip, and this peak scales with antenna gain. There is a large "sweet spot" plateau where throughput decreases and latency increases in a manner consistent with free space propagation losses. The range for each curve is proportional to the RF link gain provided by the antennas, ranging from $0 \mathrm{dBi}$ for the two dipoles to +24 $\mathrm{dBi}$ for the parabolic antenna. The "sweet spot" seems to be present in all combinations from about $80 \mathrm{~m}$ to $600 \mathrm{~m}$, where good link quality is maintained with a relatively high throughput and low latency. This testbed data can be used to determine actual propagation losses.

The Orinoco DSSS radio system throughput graph follows (Figure 7). As expected, the Orinoco outperformed the Proxim in the network throughput test with five times the throughput, corresponding to the ratio-of the raw data rates. This does not come without potential drawbacks, though. A very interesting result is a sharp increase from 150 meters to 380 meters, evocative of the Proxim result! The two radio systems are completely different in modulation and basic characteristics, and yet there is a similar variance in throughput versus distance at $20-200 \mathrm{~m}$. Both 802.11 and $802.11 \mathrm{~b}$ use similar MAC layer negotiation protocols. This is yet another clue that this inconsistency is due to timing interactions in the MAC layer. Subsequently, there is a linear decrease in throughput as distance is increased to the maximum $1.1 \mathrm{~km}$. The Orinoco product had significantly greater range than the Proxim product for reasons quantified in the next section.

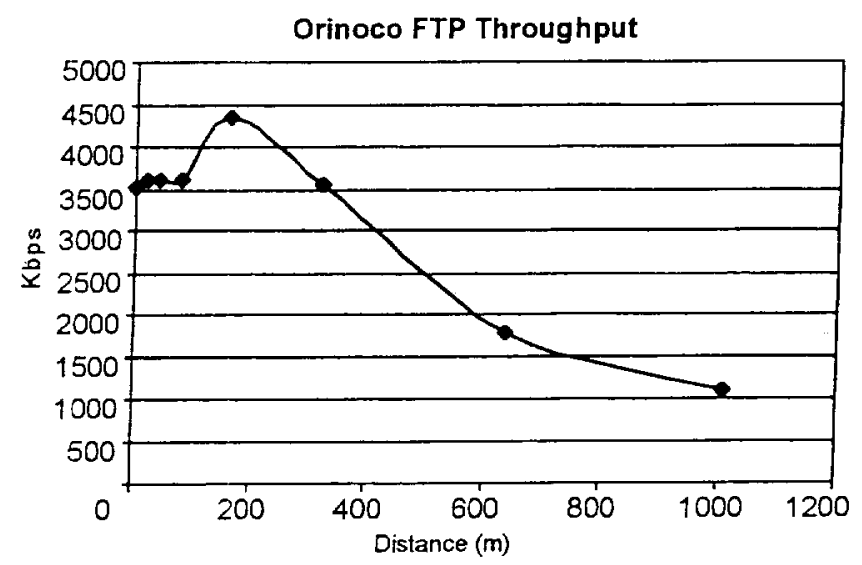

Figure 7. Orinoco FTP throughput 


\section{RF LINK ANALYSIS}

Conventional RF link analysis was applied to both radio systems using the manufacturer's specifications in order to determine theoretical operating range in a vacuum. Transmitter power, antenna gain and receiver sensitivity are used to calculate maximum range considering only free space loss. Of course, in the real world one must consider atmospheric effects and parasitic losses as well. Since these extra losses are unknown initially, one can work backward, comparing the theoretical distances to those measured in the field to determine additional losses in the field test setup. These additional losses can be further analyzed to determine if therc is a component that scales linearly with distance and which would therefore represent the effect of atmospheric water vapor. The frequencies used for these radios, $2.4 \mathrm{GHz}$, lie within the water absorption band and therefore water vapor will affect propagation of the signals on earth.

The following table demonstrates quite dramatically that the Proxim radio systems have significantly less range than predicted from free space losses alone. A major initial impetus for these tests is that the MEX field systems did not perform as well as expected and determining the cause could improve performance. However, the amount of additional loss for the Proxim system is rather high. The "link budget" represents the maximum fade margin in $\mathrm{dBm}$, and the "max range" represents the corresponding theoretical operating limit in vacuum. "Actual range" is determined by analyzing throughput versus distance graphs for each radio system.

Determining "actual range" requires inderstanding radio operation details to correctly interpret field data, and this interpretation is somewhal different for the two radio systems Many years of wireless network testing have resulted in a clear understanding of the behavior of the self-adapting modulation feature of these radios. It falls back from quad phase shift keying to bi-phase (BPSK) keying when the radio signal is too weak to support full rate data transfer. The Proxim system switches from a 1.6 Mbps rate to $800 \mathrm{Mbps}$ just before a loss of connection occurs. Estimating the point at which these rate changes occur in the field tests allows estimating the actual range.

The Proxim system has a $1.6 \mathrm{Mbps}$ raw data rate which actually delivers less than half that (typically $33 \%$ ) in sustainable throughput due to packet-- and MAC-layer overhead. Therefore the initial slope of throughput falloff is used to estimate the point at which throughput falls off below $200 \mathrm{Kbps}$. This is the point at which BPSK is no longer effective, being $25 \%$ of the raw data rate of 800 Kbps which is applied consistently to the data sets. This actual range is then used to calculate the additional loss due to all effects in the field tests. These "additional losses" (Addit'l) are very high, ranging from 16-33 dB. Further analysis attempted to identify parasitic losses and calculate atmospheric losses. Cable and connector losses were calculated from manufacturer's specifications to determine total "cable loss" for each test setup. Parasitic cable losses can be calculated readily, so there cannot be any unaccounted-for losses in the system except from atmospheric absorption. The use of long cables $(36 \mathrm{ft}$ ) to connect the Proxim AP to the 11 and $24 \mathrm{dBi}$ antennas (but not $0 \mathrm{dBi}$ dipole) added a significant extra loss of $2.6 \mathrm{~dB}$, reducing output power by nearly one-half. Furthermore the Proxim EP was connected to the ATV antenna by a six-foot length of RG-58 losing another $2 \mathrm{~dB}$ total and the calculation takes this into account. The remaining loss was divided by the actual test distance to determine additional attenuation per meter ("atmospheric loss" [Atmo]). This is about $0.02 \mathrm{~dB}$ per meter, comparable to ultra-low loss cable. Propagation of $2.4 \mathrm{GHz}$ FHSS signals through the atmosphere results in significant attenuation primarily due to absorption in water vapor.

Table 1. Proxim RF link analysis

\begin{tabular}{|c|c|c|c|c|c|c|c|c|c|}
\hline \multicolumn{10}{|c|}{ Proxim $X R$} \\
\hline & $\begin{array}{c}\text { Xmit } \\
\text { Pwr } \\
\text { (dBm) }\end{array}$ & $\begin{array}{c}\text { Recv } \\
\text { Sens } \\
(\mathrm{dBm})\end{array}$ & $\begin{array}{l}\text { Ant } \\
\text { Gain } \\
\text { (dBi) }\end{array}$ & \begin{tabular}{|l} 
Link \\
Budget \\
(dB)
\end{tabular} & $\begin{array}{c}\text { Max } \\
\text { Range } \\
\text { (m) }\end{array}$ & $\begin{array}{c}\text { Actual } \\
\text { Range } \\
\text { (m) }\end{array}$ & $\begin{array}{l}\text { Addit'l } \\
\text { Loss } \\
\text { (dB) }\end{array}$ & $\begin{array}{l}\text { Cable } \\
\text { Loss } \\
\text { (dB) }\end{array}$ & $\begin{array}{c}\text { Atmo } \\
\text { Loss } \\
(\mathrm{dB} / \mathrm{m})\end{array}$ \\
\hline \multicolumn{10}{|l|}{ Antenna } \\
\hline O dBi dipole & 27 & 85 & 0 & 112 & 3879 & 600 & 16.2 & 2.0 & 0.0237 \\
\hline $11 \mathrm{dBi}$ sect. & 27 & 85 & 11 & 123 & 13764 & 900 & 23.7 & 4.6 & 0.0212 \\
\hline 24 dBi parab. & 27 & 85 & 24 & 136 & 61482 & 1350 & 33.2 & 4.6 & 0.0212 \\
\hline
\end{tabular}


The Orinoco system was tested with only one antenna, so the RF analysis was performed on only one system configuration but in three different bandwidth regimes. The Orinoco radio has a much lower receiver sensitivity than the Proxim, $6 \mathrm{~dB}$ more sensitive at $2 \mathrm{Mbps}$. Agere provides receiver sensitivity specifications tied to raw data rate, and the Orinoco has four different data rates: 11 Mbps, 5.5 Mbps, $2 \mathrm{Mbps}$ and $1 \mathrm{Mbps}$. Using similar assumptions, one finds that only $11 \mathrm{Mbps}$ raw rate can support sustainable throughput in excess of $2.8 \mathrm{Mbps}$, only 5.5 Mbps can sustain over $1.6 \mathrm{Mbps}$ and only 2 Mops can sustain over 0.6 Mbps. Cable loss was minimized since the antennas were directly attached to the radio PC-cards. Determining actual range for each raw data rate and sensitivity, we can calculate the additional loss at a nearly constant $4.5 \mathrm{~dB}$. The atmospheric loss of the Orinoco at $11 \mathrm{Mbps}$ is half that of the Proxim system as shown in Table 2. Further analysis of the three bandwidth regimes also provides an intriguing result, that the atmospheric attenuation in each band decreases as the bandwidth decreases. Of course, this is intuitively correct, since it takes a better signal to noise ratio to correctly interpret modulation at a higher data rate. This first order approximation of linear propagation loss does not account for dispersion and other effects, but should be useful for estimating new WLAN performance characteristics.

Finally, the radio diagnostic tools provided by Agere for the Orinoco were used to create a graph of received signal level versus distance as shown in Figure 8 below.[15] At $100 \mathrm{~m}$ the level is about $-60 \mathrm{dBm}$ and at $1000 \mathrm{~m}$ it is about $-87 \mathrm{dBm}$. The difference is $27 \mathrm{~dB}$, which corresponds with $20 \mathrm{~dB}$ due to free-space loss, for a 10 to 1 distance change plus the $5 \mathrm{~dB}$ additional loss calculated above. This graph (Figure 8) is constructed directly from the radio circuits of the Orinoco calibrated in $\mathrm{dBm}$ and acts as an important cross-check for the RF link calculations.

Table 2. Orinoco RF link analysis

\begin{tabular}{|c|c|c|c|c|c|c|c|c|c|}
\hline \multirow{2}{*}{ Bandwidth } & \multicolumn{9}{|c|}{ Orinoco 0 dBi antennas } \\
\hline & $\begin{array}{c}\text { Xmit } \\
\text { Pwr } \\
(\mathrm{dBm})\end{array}$ & $\begin{array}{c}\text { Recv } \\
\text { Sens } \\
(\mathrm{dBm})\end{array}$ & $\begin{array}{l}\text { Ant } \\
\text { Gain } \\
\text { (dBi) }\end{array}$ & $\begin{array}{c}\text { Link } \\
\text { Budget } \\
\text { (dB) }\end{array}$ & $\begin{array}{l}\text { Max } \\
\text { Range } \\
\text { (m) }\end{array}$ & $\begin{array}{c}\text { Actual } \\
\text { Range } \\
\text { (m) }\end{array}$ & $\begin{array}{l}\text { Addit'l } \\
\text { Loss } \\
\text { (dB) }\end{array}$ & $\begin{array}{l}\text { Cable } \\
\text { Loss } \\
(\mathrm{dB})\end{array}$ & $\begin{array}{c}\text { Atmo } \\
\text { Loss } \\
(\mathrm{dB} / \mathrm{m})\end{array}$ \\
\hline $11 \mathrm{Mbps}$ & 15 & 83 & 0 & 98 & 774 & 450 & 4.7 & 0.2 & 0.0100 \\
\hline $5.5 \mathrm{Mbps}$ & 15 & 87 & 0 & 102 & 1227 & 750 & 4.3 & 0.2 & 0.0054 \\
\hline 2 Mbps & 15 & $\subseteq 1$ & 0 & 106 & 1944 & 1200 & 4.2 & 0.2 & 0.0033 \\
\hline
\end{tabular}

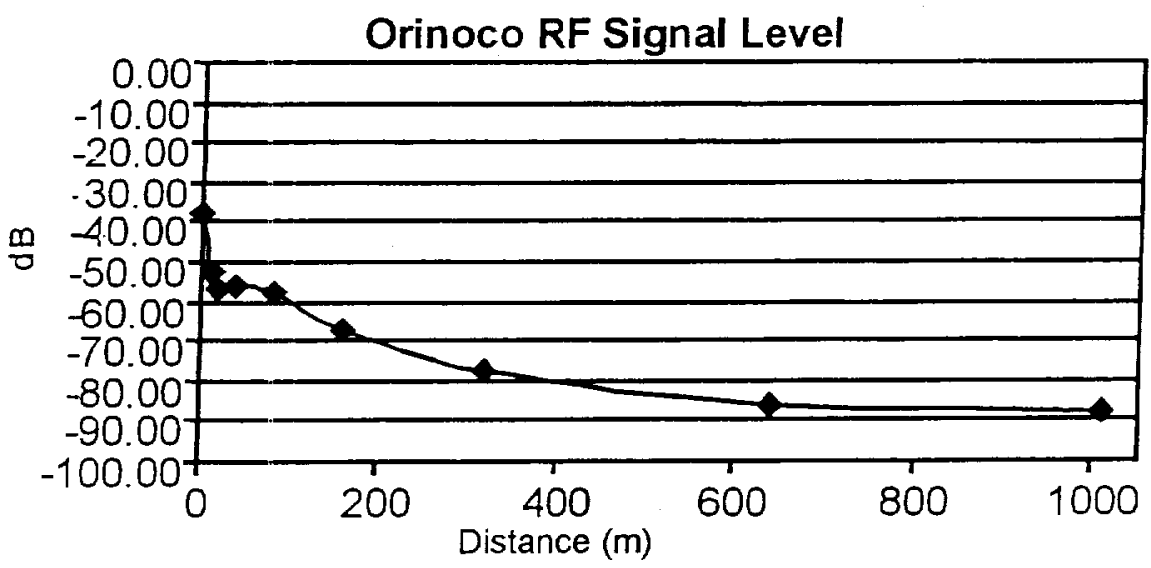

Figure 8. Orinoco Radio Signal Levels 


\section{INTERFERENCE AND SCALABILITY}

Interference caused by electromagnetic energy in the same frequency band used by WLANs to communicate data can cause a loss of throughput, increased latency and even loss of connection. The same metrics used to characterize performance can be used to study the effects of interference. Furthermore, interference effects primarily limit scaling up WLANs by using multiple co-located WLAN systems to increase aggregate bandwidth. We study the effects of interference on the two WLAN systems and attempt to layer multiple WLANs to determine scalability limits.

The interference tests were designed to stress the robustness of the data link in each radio system when subjected to a strong in-band interference source. The source used to create the interference is an analog video transmission device (Trango) with four selectable channels all within the $2.4 \mathrm{GHz}$ ISM band providing fairly high average and peak signal strengths. This source was placed between two WLAN nodes spaced about $3 \mathrm{~m}$ apart. The interference source was placed both within and outside the current working bandwidth of the WLAN system and the effect on link performance measured. Figure 9 shows the RF spectrum of the interference source, mostly in the lower part of the ISM band, obtained using the Proxim sampling spectrum analyzer.
The Proxim and Orinoco systems produce very different spectrums, as shown in Figure 10. Notice the difference between the peak (gray) and average values (black) of each bar. Each bar represents a $1 \mathrm{MHz}$ slice of the 2.40 $2.483 \mathrm{GHz}$ ISM band - 83 bars total. The Proxim produces narrow, $1 \mathrm{MHz}$-wide signals with high peak and average energies representing the frequency hops. An Orinoco running on Channel 4 produces a broad spectrum of much lower peak and average energy, putting its primary information into the small hump of average power at the lower end of the spectrum. The DSSS radio spreads its information across a larger part of the ISM band, providing higher raw data rates compared to FHSS, by putting the information into more simultaneous signal bands. The observant reader will notice an FHSS hop in the Orinoco spectrum and residual DSSS energy in the Proxim spectrum since both systems operate simultaneously in our test lab.

The interference source puts significant peak and average energy into a very limited portion of the band, which can interfere with WLAN signals in the same area. If an interference source is placed within the current operating band of the Orinoco, the entire WLAN link is lost and throughput is zero. If the interference source and WLAN channel allocation are shifted apart, then there is minimal throughput loss. The Proxim system, however does not lose the link and only about $10 \%$ of its throughput when an interference source is present, regardless of channel settings. As expected, the higher bandwidth DSSS system is much more sensitive to in-band interference.

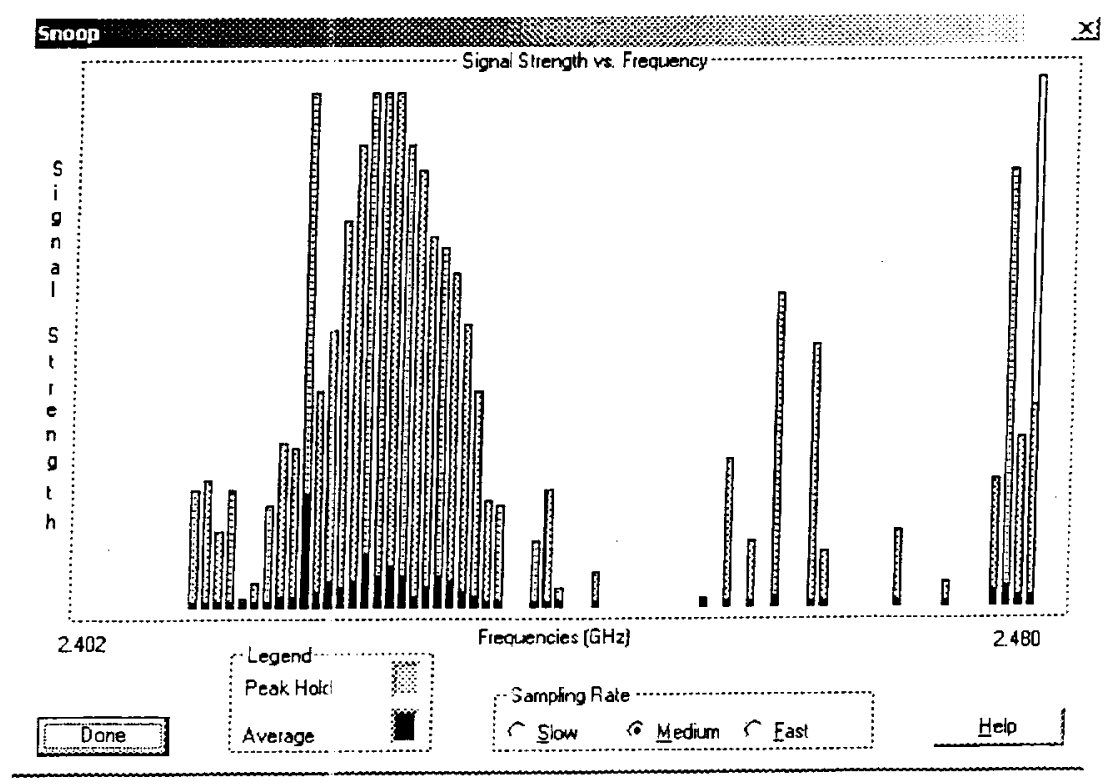

F gure 9. Interference source spectrum 

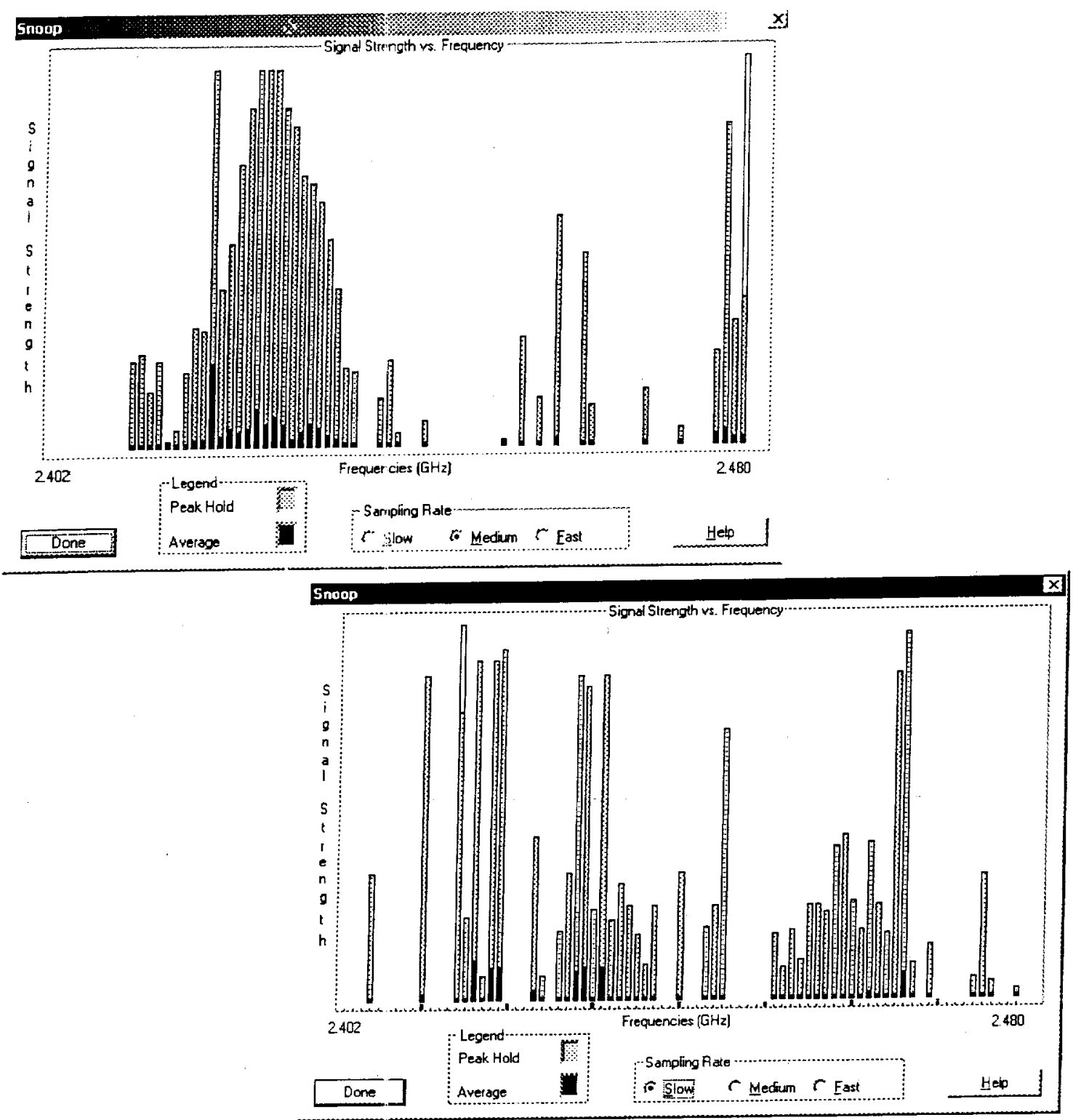

Figure 10. Orinoco Channel 4 spectrum (top) and Proxim spectrum

"Scalability" is defined as how many WLAN nodes or radio systems can be run in the same vicinity. Interference is the prime factor in salability by reducing connection reliability and by reducing throughput. Keeping interference susceptibility to a minimum allows for maximum scalability. We initially considered the Orinoco and Proxim systems individually. Proxim has developed frequency-hopping radios with fifteen different hopping sequences. Theoretically, fifteen separate networks could operate at the same time, providing up to 15 times the aggregate bandwidth of a single subnet. In reality this number is only five, and after five networks have been set up in the same vicinity, the performance of all decreases sharply. This was tested in much the same way as the in- terference tests, simply running five Proxim subnets in our lab, with each node about $3 \mathrm{~m}$ from another within the 10 by $15 \mathrm{~m}$ lab.

The Orinoco system can only support three co-located networks. Orinoco recommends that channels one, seven, and eleven be used to minimize channel overlap, which can be confirmed by looking at the Orinoco channel structure on the spectrum analyzer. This was also confirmed by tests in the lab. Notably, adding an Orinoco network does not have much affect on the Proxim system, given its much greater immunity to interference. Conversely, a Proxim system has minimal effect on an Ori- 
noco system since the Proxim spectum does not have the high average values of the interference source used previously. Our lab supports both systens running simultaneously without observing any negative interactions.

\section{CONCLUSIONS}

These experiments provided the empirical data required for beginning the design of a second generation MEX communication architecture. The experimental tools and procedures have been verified over a period of many years in a variety of environments; however, this was the first time outdoor testing was conducted under controlled conditions. Operationally, the MEX required more robust network links and higher throughput than the original Proxim system provided. These tests yielded data on what effects caused the links to perform more poorly than expected: high losses in cables and connectors, variable latency and throughput in the mid-range, and high atmospheric absorption.

Although cables were constructed according to accepted practice using low-loss cable suitable for the frequency range, losses at $2.4 \mathrm{GHz}$ are double those for $1 \mathrm{GHz}$ frequencies. This large factor requires significant changes in conventional engineering practices when dealing with WLAN technology. For example, the radio units should be mounted on the repeater mast, siace this is the only way to reduce the cable length and hence losses. This complicates the mechanical and electrical design of the repeater unit. These issues are particularly important for WLANs since they use low-power transmitters which cannot tolerate additional losses. Furthermore, as WLAN frequencies and bandwidths increase. these factors will become even more important

Other WLAN performance issues are related to the serious variations in throughput that were observed at medium range, hypothetically due to $\mathrm{MAC}$ layer timing interactions. These variations manifested themselves as poor performance of WLAN links near base camp and could not be explained on the basis of RF signal levels, which were high. Rather, the performance variations resulted from small changes in mobile ATV position and even as changes in link performance over time. This could not be explained prior to creating the calibration curves which showed they are due to unusually high latencies under certain mid-range conditions.

A major surprise of the testing was the difference between FHSS and DSSS radios in regard to atmospheric absorption. The data show a two-to-one difference between the Proxim and Orinoco at full bandwidth (1.6 Mbps vs 11 $\mathrm{Mbps})$ and an eight-to-one difference at similar bandwidth. This particular effect was too pronounced to be attributable to experimental error. The Proxim system can not cover $1 \mathrm{~km}$ without a directional antenna, unlike the
Orinoco. It is speculated that the higher power levels of the FHSS radio cause greater excitation of water molecules and therefore greater absorption and that this effect is not linear. However, the DSSS radio's ability to cover greater range at greater bandwidth has significant tradeoffs. Interference testing clearly demonstrated increased susceptibility to in-band interference, which translates into increased susceptibility to multipath interference caused by walls, hills or any other obstruction. Therefore, in hilly terrain, the DSSS may not have a real range advantage since it is more likely to suffer degradation due to multipath.

Finally, the two different radio systems scale differently. Five Proxim subnets would deliver $8 \mathrm{Mbps}$ of aggregate raw data bandwidth while three Orinoco subnets would deliver $33 \mathrm{Mbps}$. The $2.4 \mathrm{GHz}$ ISM band provides a total of $83 \mathrm{MHz}$ of bandwidth: neither radio system fills this completely. There are improvements possible in providing higher bandwidth. One practical approach is to run FHSS and DSSS systems concurrently, providing a balance of desirable characteristics. We will attempt to determine scalability of mixed mode WLANs in future tests

Successful design of WLAN systems requires the communications engineer to have technical competence in both the radio and computer network domains - a challenging role. Radio analysis is required to provide adequate link margins, and network expertise is required to understand radio subnet setup, MAC layer tuning and overall architecture. The analytical technique for lumping all atmospheric effects, including wave dispersion, into one linear constant is useful for estimating the performance of new designs. Future work will include design and test of a hybrid communication system incorporating the best aspects of both FHSS and DSSS systems. MAC layer timing interactions should be further explored.

\section{REFERENCES}

[1] Wireless LAN Association. What is a Wireless LAN? [http://www.wirelesslan.com/wireless/] February, 1999.

[2] R. Alena, Wireless Network Experiment-Risk Mitigation Experiment 1306, report on STS-74/76-Mir20 experiment for the Phase One International Space Station Program, 1996.

[3] R. Alena, E. Yaprak, and S. Lamouri, "Modeling A Wireless Network For International Space Station," IEEE Aerospace Conference, 1999. 
[4] K. Feher. Wireless Digital Commanications: Modulation and Spread Spectrum Applications. Prentice Hall, April, 1995.

[5] W.E. Lutz. Wireless LAN Technologies. [http://icensedinfo.s.nsa/FACCTs/00014337.htm]. January, 2000.

[6] J. Geier, Wireless LANs: Implemeinting Interoperable Networks, (pp. 93-131), Macmillan Technical Publishing, 1999.

[7] NASA Human Exploration and D:velopment of Space [http://www.hq.nasa.gov/osf/heds/]. August, 2001.

[8] Stephen Braham, Peter Anderson, Pascal Lee, Richard Alena, and Brian Glass, "Canada and Analogue Sites for Mars Exploration," Proceedings of the Second Canadian Space Exploration Workshop, 1999.

[9] Stephen P. Braham, Richard Alena Bruce Gilbaugh, and Brian Glass, "Space Networking and Protocols for Planetary Exploration and Analog Planetary Sites," IEEE Aerospace Conference, 2001.

[10] R. Alena, B. Gilbaugh, and B. Glass, "Communication System Architecture for Planetary Exploration," IEEE Aerospace Conference, 2001.

[11] Proxim Corporation RangeLAN2' 'echnical Reference Guide. Proxim, Inc. USA, [bttp://www proxim com], 2000.

[12]BreezeCOM. Wireless LAN Concepts: Appendix B [http://www.Breezecom.com/TechSupport/bnpro-ab.htm]. August, 2000.

[13] W. Tomasi. Electronic Communicaiions Systems: Fundamentals Through Advanced. Prenice Hall, USA, 1998.

[14] Bruce L. Gilbaugh, Brian Glass, and Richard Alena (2001) "Mobile Network Field Testing at HMP-2000," IEEE Aerospace Conference, 2001.

[15] Orinoco User's Manual

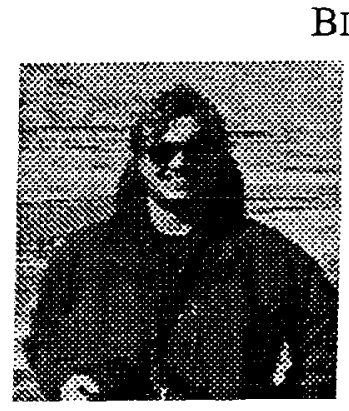

Richard Alena is a Computer Engineer and the Group Lead for the Intelligent Mobile Technologies (IMT) Lab and the Mobile Exploration System testbed at NASA Ames Research Center. The IMT team integrates mobile hardware and software components into unique systems supporting human performance for flight and payload operations aboard spacecraft. He was principal investigator for the Wireless Network Experiment flown aboard Shuttle and Mir, technology later adopted by the International Space Station Program. Rick spent three summers in the Canadian Arctic developing mobile technologies for human planetary exploration. He has a MSEE\&CS from University of California, Berkeley.

Darin Evenson (below left) is a Lieutenant in the United States Navy and a graduate student at the Naval Postgraduate School (NPS) in Monterey, California. He is interested in applying mobile wireless technologies to small groups of people deployed in hostile environments. He completed his undergraduate work at the United States Naval Academy and has recently graduated from NPS with a MS in Information Technology Management and an MS in Space Systems Operations.
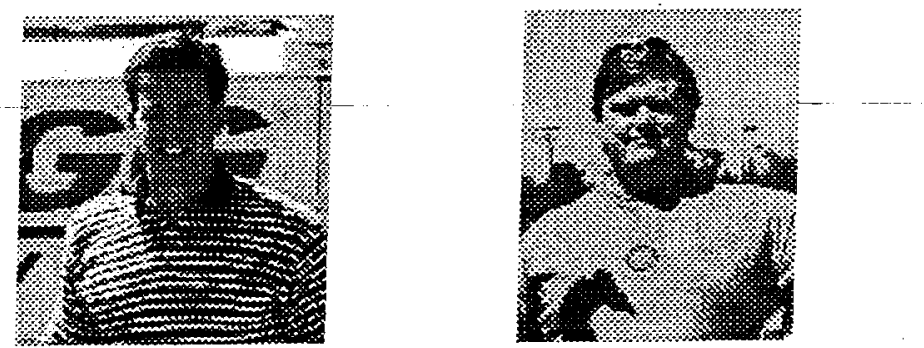

Victor Rundquist (above right) spent two years working as a master electrician for a sound and lighting company in Phoenix, AZ and later went on to manage a retail electronics store. Victor has been attending De Anza College for three years taking courses in advanced mathematics and the Physical Sciences and will be graduating in 2005 with a degree in Electrical Engineering and Computer Science. He has been working at NASA/Ames Research Center in the Intelligent Mobile Technologies group for the past year, through the Foothill-De Anza College Internship Program. 